\title{
Associative, bidirectional changes in neural signaling utilizing NMDA receptor- and endocannabinoid- dependent mechanisms
}

\author{
Qin Li and Brian D. Burrell ${ }^{1}$ \\ Neuroscience Group, Division of Basic Biomedical Sciences, Sanford School of Medicine at the University of South Dakota, \\ Vermillion, South Dakota 57069, USA
}

\begin{abstract}
Persistent, bidirectional changes in synaptic signaling (that is, potentiation and depression of the synapse) can be induced by the precise timing of individual pre- and postsynaptic action potentials. However, far less attention has been paid to the ability of paired trains of action potentials to elicit persistent potentiation or depression. We examined plasticity following the pairing of spike trains in the touch mechanosensory neuron ( $\mathrm{T}$ cell) and $\mathrm{S}$ interneuron ( $\mathrm{S}$ cell) in the medicinal leech. Long-term potentiation (LTP) of T to $S$ signaling was elicited when the T-cell spike train preceded the S-cell train. An interval 0 to +1 sec between the T- and S-cell spike trains was required to elicit long-term potentiation (LTP), and this potentiation was NMDA receptor (NMDAR)-dependent. Long-term depression (LTD) was elicited when S-cell activity preceded T-cell activity and the interval between the two spike trains was $-0.2 \mathrm{sec}$ to $-10 \mathrm{sec}$. This surprisingly broad temporal window involved two distinct cellular mechanisms; an NMDAR-mediated LTD (NMDAR-LTD) when the pairing interval was relatively brief $(<-1 \mathrm{sec}$ ) and an endocannabinoid-mediated LTD (eCB-LTD) when longer pairing intervals were used ( -1 to $-10 \mathrm{sec}$ ). This eCB-LTD also required activation of a presynaptic transient receptor potential vanilloid (TRPV)-like receptor, presynaptic $\mathrm{Ca}^{2+}$ release from intracellular stores and activation of voltage-gated $\mathrm{Ca}^{2+}$ channels (VGCCs). These findings demonstrate that the pairing of spike trains elicits timing-dependent forms of LTP and LTD that are supported by a complex set of cellular mechanisms involving NMDARs and endocannabinoid activation of TRPV-like receptors.
\end{abstract}

[Supplemental material is available for this article.]

Associative forms of long-term neuroplasticity, as first proposed by Hebb (1949), depend on the temporal relationship between pre- and postsynaptic activity. Studies of spike-timing-dependent plasticity (STDP) have provided experimental confirmation of this principle, specifically that in most connections long-term potentiation (LTP) is produced when a presynaptic action potential (or spike) precedes the postsynaptic spike, whereas long-term depression (LTD) is produced when the order is reversed (see review by Caporale and Dan 2008). In addition to the temporal order being important in determining the sign of the synaptic change, temporal proximity is also critical in that the time between the pre- and postsynaptic activity must be sufficiently brief, usually $<40 \mathrm{msec}$, to support potentiation or depression.

It has been noted that the time intervals between pre- and postsynaptic activity that support STDP are not as long as those observed in many associative learning experiments, in which interstimulus intervals on the scale of seconds can be used to elicit successful conditioning (Drew and Abbott 2006). Most studies of STDP involve pairing single pre- and postsynaptic spikes or brief bursts of two to three spikes; however, many neurons communicate using relatively long trains of action potentials that are tens to hundreds of milliseconds long. The use of spike trains to elicit LTP and LTD may allow for longer intervals between pre- and postsynaptic activity than observed during STDP and be more similar to the temporal windows observed in behavioral experiments in which learning and memory are produced.

\footnotetext{
'Corresponding author.
}

E-mail bburrell@usd.edu.

Article is online at http://www.learnmem.org/cgi/doi/10.1101//m.2252511.
In this study, we used the central nervous system (CNS) of the medicinal leech to examine long-term modulation of neuronal signaling following the pairing of spike trains between the touch mechanosensory neurons (T cells) and the $\mathrm{S}$ interneuron (S cell). The T cell is a low-threshold mechanosensitive neuron with input to a number of neurons that mediate motor withdrawal or escape behaviors, while the $\mathrm{S}$ cell is thought to be important for learning and arousal in the leech whole-body shortening reflex (Sahley et al. 1994; Modney et al. 1997; Burrell et al. 2003; Kristan et al. 2005; Crisp and Burrell 2008). The T to S synaptic circuit (Fig. 1A) consists of both a monosynaptic electrical component and a polysynaptic, glutamatergic component (Muller and Scott 1981; Li and Burrell 2008), although the identities of the interneurons that mediate the glutamatergic component are not known. Previous studies have shown that this synaptic circuit can undergo LTP and LTD as a result of tetanic stimulation and low-frequency stimulation (LFS), respectively (Burrell and Sahley 2004; Burrell and Li 2008; Li and Burrell 2009), but it was unknown whether pairing of T- and S-cell activity results in persistent changes in synaptic signaling.

In this paper, both LTP and LTD were observed following pairing spike trains that mimic the physiologically relevant patterns of T- and S-cell activity during whole-body shortening. The pattern of this plasticity was similar to that observed during STDP; LTP following presynaptic-before-postsynaptic pairings and LTD following postsynaptic-before-presynaptic pairings. One significant difference was the intervals between the T- and S-cell spikes trains that supported LTP and LTD were on the order of hundreds of milliseconds to seconds, with the temporal window governing LTD being especially broad. LTP was induced by 
A
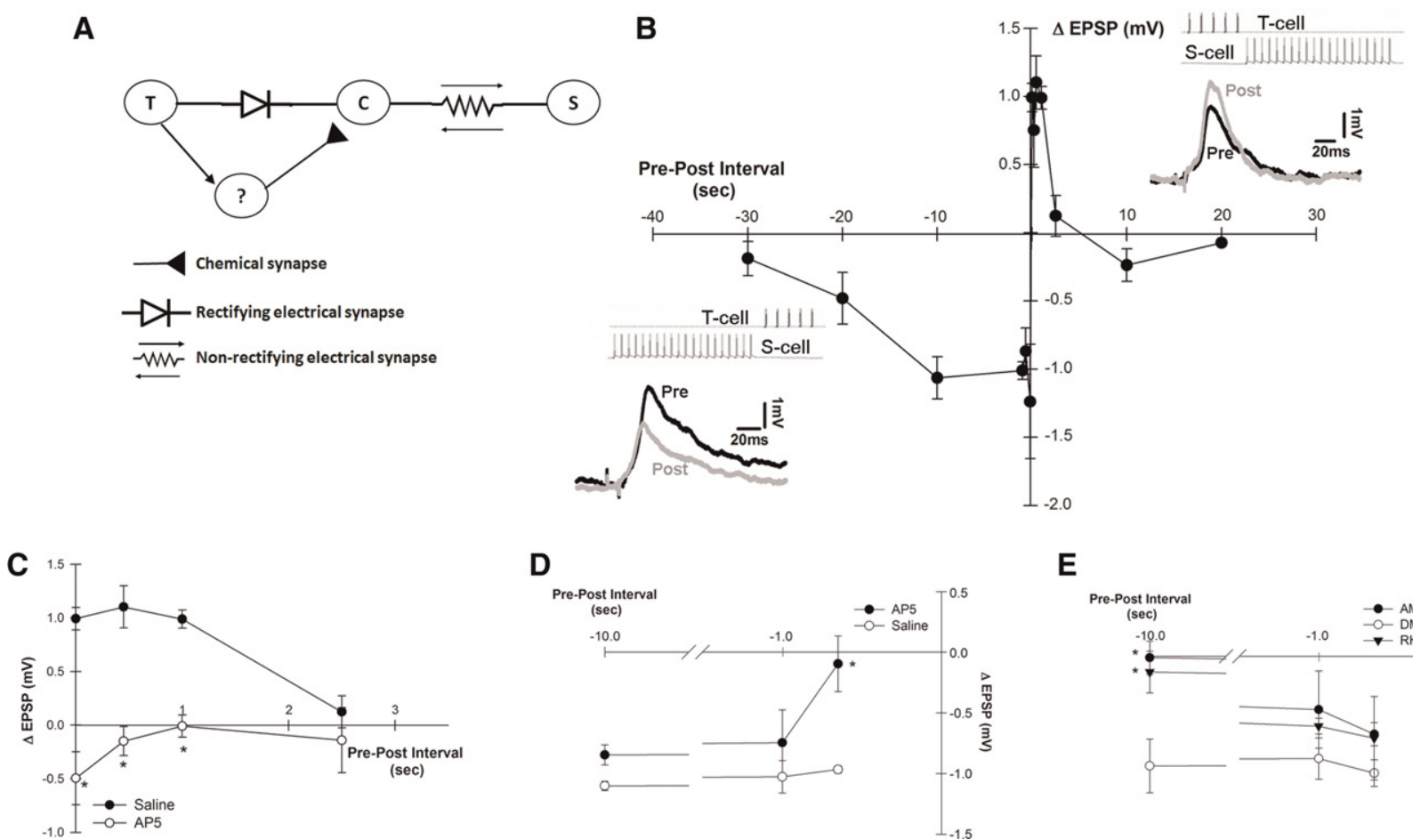

D

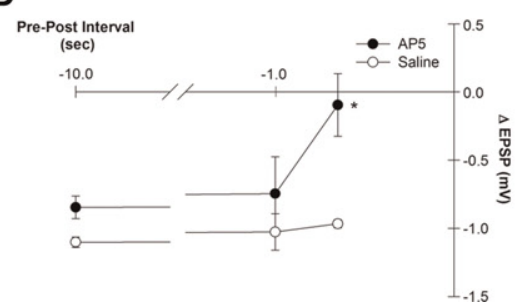

E

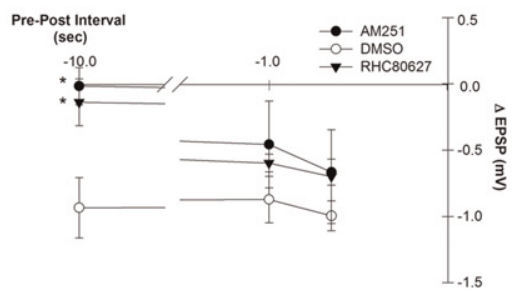

Figure 1. (A) Diagram of $T$ to $S$ synaptic circuit. T-cell synaptic input to the $S$ cell is routed through the coupling (C) interneuron which has a nonrectifying electrical synapse with the S cell and a rectifying electrical synapse with the T cell (Muller and Scott 1981). As a consequence of the strong electrical coupling between the $S$ and $C$ cells, excitatory presynaptic potentials (EPSPs) initiated in the $C$ cell undergo little or no attenuation or delay when they arrive in the S cell (Muller and Scott 1981). The identity of the interneuron(s) that mediates the chemical (glutamatergic) (Li and Burrell 2008) component of the polysynaptic pathway is not known ("?"). Whether the connection between the T and unknown interneuron (indicated by an arrow) is electrical or chemical is also unknown, but this pathway may be an electrical synapse (see Discussion). (B) Temporal window for LTP and LTD. Simultaneous pairings of T- and S-cell spikes trains or T before $S$ pairings $\leq 1 \mathrm{sec}$ produced LTP. Negative (S before T) pairings of -0.2 sec to -10 sec produced LTD. Insets: T- and S-cell spike trains during $T$ before $S$ and $S$ before T pairings. Also shown are pretest (black trace) and post-test (gray trace) EPSPs from LTP and LTD experiments. (C) AP5 blocked LTP following pairings with a pre-post interval of $0,0.45$, and 1.0 sec. (D) AP5 blocked LTD following pairings with a pre-post interval of $-0.65 \mathrm{sec}$, but had no effect on depression following pairings of -1 or $-10 \mathrm{sec}$. (E) Both RHC 80267 and AM251 blocked LTD following pairings of $-10 \mathrm{sec}$, but had no significant effect on depression following pairings of -1 or $-0.65 \mathrm{sec}$. Asterisks indicate significant difference compared with vehicle control.

NMDA receptor (NMDAR)-dependent mechanisms, whereas LTD was induced two distinct mechanisms, an NMDAR-dependent process when $\mathrm{S}$ before $\mathrm{T}$ interval was brief $(<1 \mathrm{sec})$ and an endocannabinoid-dependent process when the $\mathrm{S}$ before $\mathrm{T}$ interval was relatively long (between 1 and $10 \mathrm{sec}$ ).

\section{Results}

\section{Timing-dependent LTP and LTD in the T to $S$ circuit}

Changes in $\mathrm{T}$ to $\mathrm{S}$ signaling were observed following pairing of physiologically appropriate levels of activity based on the order and temporal proximity of the T- and S-cell spike trains. Both T-cell (five spikes at $10 \mathrm{~Hz}$ ) and S-cell (20 spikes at $25 \mathrm{~Hz}$ ) spike trains were consistent with the activity observed in these neurons during whole-body shortening (Shaw and Kristan 1995; Burrell and Sahley 2005). As shown in Figure 1B, potentiation was observed when the start of the $\mathrm{T}$ - and S-cell spike trains coincided ( 0 sec $\mathrm{T}-\mathrm{S}$ spike train interval) and when the interval between the T- and S-cell spike trains was $0.45 \mathrm{sec}$ and $1.0 \mathrm{sec}$ when compared with unstimulated controls (mean \pm SE of $\Delta$ excitatory postsynaptic potential [EPSP] in controls $=-0.09 \pm 0.07 \mathrm{mV} n=5$; one-way analysis of variance [ANOVA], $F_{(6,18)}=3.11, P<0.001$; Newman-Keuls post hoc test, $P<0.01$ ). No changes in T to $\mathrm{S}$ signaling were observed following pairings of $2.5,10$, and 20 sec. LTD was produced when S-cell activity precedes T-cell activity.
Surprisingly, the LTD temporal window was even broader with depression being observed following pairing with intervals of -0.2 (spike trains overlapped), $-0.65,-1$, and -10 sec (Fig. 1B; one-way ANOVA, $F_{(5,24)}=4.17, P<0.01$; Newman-Keuls post hoc test, $P<0.001$ ). Pairings of -20 sec produced a modest level of depression that was not statistically significant from the unstimulated control group, while pairings of $-30 \mathrm{sec}$ produced no change in $\mathrm{T}$ to $\mathrm{S}$ signaling. No changes were observed in the electrical component of the T to S EPSP at any of the pairing intervals tested (Supplemental Fig. 2A).

\section{Timing-dependent LTP was mediated by NMDA receptors} The potential role of NMDARs during LTP following spike train pairing was examined using the selective NMDAR antagonist AP5. As shown in Figure 1C, AP5 $(20 \mu \mathrm{M})$ blocked LTP induced by pairing protocols 0 sec (one-way ANOVA. $F_{(1,8)}=39.99, P<$ 0.001 ), $+0.45 \mathrm{sec}$ (one-way ANOVA, $F_{(1,8)}=25.29, P<0.001$ ), and +1 sec (one-way ANOVA, $F_{(1,8)}=47.64, P<0.001 ; n=5$ in each experimental group). AP5 had no effect on T to $S$ connections that underwent +2.5 -sec pairing (Fig. 1C; $n=5$ in each group, one-way ANOVA, $\left.F_{(1,8)}=0.03, P>0.05\right)$ and AP5 application did not affect the synaptic transmission in a set of preparations in which activity was omitted (data not shown), consistent with earlier AP5 control studies with this synapse (Burrell and Sahley 2004; Li and Burrell 2009). These results agree with 
previous studies of NMDAR-LTP in the leech pressure $(\mathrm{P})$ to anterior pagoda (AP) cell synapse following pairing or pre- and postsynaptic spike trains (Grey et al. 2009; Grey and Burrell 2010). Since these previous studies examined in detail the cellular mechanisms mediating LTP following pairing of pre- and postsynaptic spike trains, attention in the current study was focused on the properties of pairing-dependent LTD given the broad temporal window that supports this form of plasticity.

\section{Timing-dependent LTD was mediated by multiple cellular mechanisms}

The role of NMDARs during LTD elicited by negative pairing ( $\mathrm{S}$ before $\mathrm{T}$ ) was also tested. Although depression was observed following pairing interval of $-0.2 \mathrm{sec}$, this interval was not further tested because the T- and S-cell spike trains overlap and any resulting depression is likely the result of both STDP processes (where the spike trains overlap) and spike train pairing-dependent processes. As shown in Figure 1D, AP5 $(20 \mu \mathrm{M})$ prevented the induction of LTD when the $\mathrm{T}-\mathrm{S}$ pairing interval was at -0.65 sec (one-way ANOVA, $F_{(1,10)}=$ $12.4, P<0.05 ; n=6$ in each group), but did not significantly block depression induced by longer negative pairing intervals, that is at -1 sec (one-way ANOVA, $F_{(1,8)}=1.13, P>0.05 ; n=5$ in each group) and -10 sec (one-way ANOVA, $F_{(1,10)}=1.02, P>0.05 ; n=5$ in each group). These results indicated that while NMDARs contributed to LTD when the $S$ before $T$ interval was relatively brief, other cellular processes must mediate LTD following pairing with longer pairing intervals.

To confirm the AP5 results and to determine the location of NMDARs mediating LTD following pairing with the -1-sec negative interval, MK-801 (5 mM), an open channel blocker of NMDA receptor was injected into either the T or S cell. MK801 injection into $S$ cell, but not the T cell, inhibited the LTD following pairing with the -0.65 -sec T-S interval indicating that this pattern of pre- and postsynaptic activity required activation of postsynaptic NMDARs to induce depression (Fig. 2A; one-way ANOVA, $F_{(2,12)}=$ 7.49, $P<0.01$; Newman-Keuls post hoc test, $P<0.01$ between MK801 in $S$ and saline; $n=5$ for each group). When LTD following pairing with a -10 -sec $T$-S interval was carried out, neither T- nor S-cell MK801 injection had any effect on the resulting depression (Fig. 2A; one-way ANOVA, $F_{(2,12)}=0.12, P>0.05 ; n=5$ for each group), agreeing with the conclusion that NMDAR activation was selectively involved LTD following short- but not longinterval pairing.

In an earlier study, T to S LTD induced by LFS was found to utilize two distinct cellular mechanisms depending on the duration of the LFS. An NMDAR-dependent depression when the LFS duration was $450 \mathrm{sec}$ and a cannabinoid-dependent LTD when the LFS duration was $900 \mathrm{sec}$ ( $\mathrm{Li}$ and Burrell 2009). Therefore, the possibility that endogenous cannabinoid transmitters (endocannabinoids) might contribute to NMDAR-independent LTD following $\mathrm{S}$ before $\mathrm{T}$ pairing was examined. As is the case for the mammalian brain, 2-arachidonoyl glycerol (2-AG) is the most abundant endocannabinoid in the leech CNS (Matias et al. 2001). Therefore, during pairing the ganglia were treated with RHC80267, an inhibitor of diacylglycerol lipase (DAGL) which is required for inhibition of 2-AG synthesis (Stella et al. 1997; Di Marzo et al. 1999). Exogenous application of 2-AG has been observed to depress multiple synapses in the leech, including $\mathrm{T}$ to S, and RHC80267 has been used to inhibit endocannabinoiddependent LTD induced by LFS in the leech (Li and Burrell 2009, 2010; Yuan and Burrell 2010a). Consistent with these earlier observations, perfusion of RHC80267 $(100 \mu \mathrm{M})$ during pairing blocked the LTD elicited by $\mathrm{T}-\mathrm{S}$ pairing interval of $-10 \mathrm{sec}$ (Fig. 1E; one-way ANOVA, $F_{(2,12)}=6.59, P<0.05$; Newman-Keuls post hoc test, $P<0.05$ between RHC80267 and saline group; $n=5$ for each group). RHC80267 did not prevent depression following -0.65 -sec pairing (Newman-Keuls post hoc test, $P>0.05$ between RHC80267 and saline group; $n=5$ ). In the case of depression following -1-sec pairing, RHC80267 appears to have partially inhibited LTD, but this effect was not statistically significant when compared with saline-treated group, possibly because LTD following this pairing interval is mediated by a combination of NMDARand endocannabinoid-dependent processes.

To further confirm the role of 2-AG during LTD following long, but not short, interval pairing and to determine the location of 2-AG synthesis, tetrahydrolipstatin (THL; $10 \mu \mathrm{M}$ ), a more selective DAG lipase inhibitor (Ortar et al. 2008), was injected into either the $\mathrm{T}$ or the $\mathrm{S}$ cell prior to pairing. As shown in Figure $2 \mathrm{~B}$, THL delivery into the $S$ cell, but not the $\mathrm{T}$ cell, prevented the induction of LTD following - 10-sec pairing (one-way ANOVA, $F_{(2,10)}=17.63, P<0.001$; Newman-Keuls post hoc test, $P<0.01$ between THL and DMSO group; $n=5$ in DMSO group). THL 
injection into either T or $S$ cell did not affect the induction of LTD following -0.65 -sec pairing interval group (Fig. 2B; one-way ANOVA, $F_{(2,9)}=1.65, P>0.05 ; n=4$ for each group). These data agree with the earlier RHC80267 results (Fig. 1E) and demonstrate that the postsynaptic $S$ cell was the site of 2-AG synthesis during this form of LTD.

As a final test that endocannabinoid signaling contributes to LTD in the T to $\mathrm{S}$ circuit, the ability of the AM251 $(10 \mu \mathrm{M})$, a CB receptor antagonist, was also examined. Although protostomal invertebrates lack orthologs for the vertebrate cannabinoid receptors CB1 and CB2 (Elphick and Egertova 2005), agonists and antagonists for these receptors surprisingly exhibit cannabinoidspecific efficacy suggesting crossreactivity between vertebrate and invertebrate cannabinoid receptors (McPartland et al. 2006; Lemak et al. 2007; Rawls et al. 2007; Li and Burrell 2009; Yuan and Burrell 2010a). In the leech, AM251 has been effective at preventing both LFS-induced synaptic depression and depression elicited by exogenous application of 2-AG (Li and Burrell 2009; Yuan and Burrell 2010a). Perfusion of AM251 during pairing (Fig. 1E) did block LTD following pairing with an T-S interval of $-10 \mathrm{sec}$ (Newman-Keuls post hoc test between AM251 and saline group $P<0.05)$ and had no effect on depression following pairing with an interval $-0.65 \mathrm{sec}$ (one-way ANOVA, $F_{(2,9)}=0.96, P>$ $0.05 ; n=4$ in each group). AM251 treatment reduced the level of depression following pairing with an T-S interval of $-1 \mathrm{sec}$, but this effect was not statistically significant when compared with the saline group (one-way ANOVA, $F_{(2,12)}=1.11, P>0.05$; $n=5$ in each group). The AM251 results are identical to the effects of RHC80267 and, along with the THL experiment, support the conclusion that an endocannabinoid-dependent modulation mediated LTD following certain patterns of pre- and postsynaptic activity in this circuit. Together, the NMDAR and endocannabinoid data demonstrate the presence of two distinct cellular mechanisms mediating LTD at the T to S connection; an NMDAR-dependent mechanism that is selectively activated during $\mathrm{T}-\mathrm{S}$ pairing with a relatively brief negative interval $(-0.65$ sec) and an endocannabinoid-dependent LTD that is selectively activated during $\mathrm{T}-\mathrm{S}$ pairing with a longer negative interval $(-10 \mathrm{sec})$.

\section{Endocannabinoid-dependent LTD was mediated by a} transient receptor potential vanilloid (TRPV)-like receptor If protostomal invertebrates, such as the leech, lack orthologs to the vertebrate $\mathrm{CB} 1$ and $\mathrm{CB} 2$ receptors, then what receptors are 2-AG and AM251 binding to? Endocannabinoids, including 2-AG, are known to also bind to TRPV 1 (TRPV1) receptors (Di Marzo et al. 2001; Golech et al. 2004; Qin et al. 2008) and TRPV1 receptors have been shown to mediate endocannabinoiddependent LTD in the mammalian brain (Gibson et al. 2008; Maione et al. 2009; Chavez et al. 2010; Grueter et al. 2010). Furthermore, a TRPV-like receptor appears to mediate ecLTD of a nociceptive synapse in the leech (Yuan and Burrell 2010a), so the possibility a TRPV-like receptor mediated endocannabinoiddependent LTD in the T to $\mathrm{S}$ circuit was examined. As shown in Figure 2C, perfusion of the TRPV antagonist capsazepine $(10 \mu \mathrm{M})$ during pairing blocked LTD following -10 -sec pairing (Fig. 2C; one-way ANOVA, $F_{(1,8)}=14.43, P<0.05 ; n=5$ in each group), but had no effect on depression following -0.65 -sec pairing (one-way ANOVA, $F_{(1,8)}=0.14, P>0.05 ; n=5$ in each group). These results are consistent with a leech TRPV-like receptor mediating endocannabinoid-dependent LTD given that capsazepine only blocked depression that was sensitive to other cannabinoidspecific drugs (RHC80267, THL, and AM251) and did not affect NMDAR-dependent LTD.
To examine the location of TRPV-like receptor, capsazepine $(100 \mu \mathrm{M})$ was injected into either the T or S cell prior to pairing (the higher concentration was to account for dilution within the injected neuron; see Gibson et al. 2008; Yuan and Burrell 2010a). Capsazepine injection into the T cell prevented the induction of eCB-LTD following the long interval pairing, as shown in Figure 2D (one-way ANOVA, $F_{(2,12)}=14.5, P<0.05$; NewmanKeuls post hoc test, $P<0.01$ between the DMSO and capsazepine treated groups; $n=5$ for each group). Capsazepine injection into the $S$ cell, however, had no affected on eCB-LTD following -10 -sec pairing (Newman-Keuls post hoc test $P>0.05$ between the DMSO and capsazepine treated groups, $n=5$ for each group). Injection of capsazepine into the T $(n=5)$ and $S$ cell $(n=4)$ without pairing (control in Fig. 2D) did not have any effect on the $\mathrm{T}$ to $\operatorname{S}$ EPSP $\left(n=5\right.$ in DMSO control; one-way ANOVA, $F_{(2,11)}=0.25$, $P>0.05)$. These results suggest that eCB-LTD required the activation of a TRPV-like receptor located on the presynaptic neuron.

\section{Role of $\mathrm{Ca}^{2+}$ signaling in during different forms of LTD}

Injections of the $\mathrm{Ca}^{2+}$ chelator EGTA $(1 \mathrm{mM})$ into the T and $\mathrm{S}$ cells were used to assess the role of intracellular $\mathrm{Ca}^{2+}$ signaling during LTD elicited by either the short $(-0.65 \mathrm{sec})$ or long $(-10 \mathrm{sec})$ interval pairing. As shown in Figure 3A, EGTA injected into either the T- $(n=6)$ or the S-cell $(n=5)$ blocked LTD following short interval $(-0.65 \mathrm{sec})$ pairing compared with the saline-injected group $(n=5)$ indicating that the increase of intracellular $\mathrm{Ca}^{2+}$ level in both neurons was necessary for this NMDAR-dependent form of depression (one-way ANOVA, $F_{(2,13)}=12.64, P<0.001$; Newman-Keuls post hoc test, $P<0.01$ between EGTA in the T cell and saline group; $P<0.01$ between EGTA in the $\mathrm{S}$ cell and saline group). LTD following the long interval $(-10 \mathrm{sec})$ pairing was also blocked by EGTA injected into either the T- $(n=7)$ or $\mathrm{S}$ cell $(n=7)$ (Fig. 3A; one-way ANOVA, $F_{(2,16)}=4.67, P<0.05$; Newman-Keuls post hoc test, $P<0.05$ between EGTA in the T cell and saline group $(n=5) ; P<0.05$ between EGTA in the $S$ cell and saline group). T- or S-cell EGTA injection without pairing had no effect on the T to S EPSP when tested at the same time point when a post-pairing test of the synapse would occur (Fig. 3A; one-way ANOVA, $F_{(2,12)}=0.104, P>0.05 ; n=5$ in each control group). These results demonstrated that both forms of LTD required increases in intracellular $\mathrm{Ca}^{2+}$ in the T and S cells.

Increased cytosolic $\mathrm{Ca}^{2+}$ required for both forms of depression may be the result of an influx of extracellular $\mathrm{Ca}^{2+}, \mathrm{Ca}^{2+}$ release from intracellular stores, or both. Sources for extracellular $\mathrm{Ca}^{2+}$ influx include NMDARs and voltage-gated $\mathrm{Ca}^{2+}$ channels (VGCCs). Previous experiments have shown that NMDARs contribute to LTD following the short, but not long interval pairing. Therefore, the role of VGCCs was tested during both forms of LTD using $1 \mathrm{mM} \mathrm{NiCl} 2\left(\mathrm{Ni}^{2+}\right)$, which has been shown to block low threshold, but not high threshold VGCCs in leech neurons (Ivanov and Calabrese 2006b). As shown in Figure 3B, $\mathrm{Ni}^{2+}$ inhibited LTD following long interval pairing (one-way ANOVA, $F_{(1,8)}=$ $19.07, P<0.01 ; n=5$ in each group) consistent with earlier studies indicating that $\mathrm{Ca}^{2+}$ influx through VGCCs contributed to eCB-LTD in the leech (Li and Burrell 2010). $\mathrm{Ni}^{2+}$ treatment did not inhibit LTD following short interval pairing (one-way ANOVA, $F_{(1,13)}=2.14, P>0.05 ; n=5$ in each group).

The potential role of $\mathrm{Ca}^{2+}$ release from intracellular stores was examined by perfusing the ganglion with thapsigargin $(1 \mu \mathrm{M})$, an inhibitor of $\mathrm{Ca}^{2+}$ ATPase, during $-0.65-\mathrm{sec}$ or -10 -sec interval pairing. As shown in Figure 3C, thapsigargin did block LTD following -10 -sec interval pairing (one-way ANOVA, $F_{(2,12)}=10.83, P<0.01$; Newman-Keuls post hoc test, $P<0.05 ; n=5$ in each group), but had no effect on LTD following -0.65 -sec interval pairing (one-way ANOVA, $F_{(2,12)}=0.23$, 

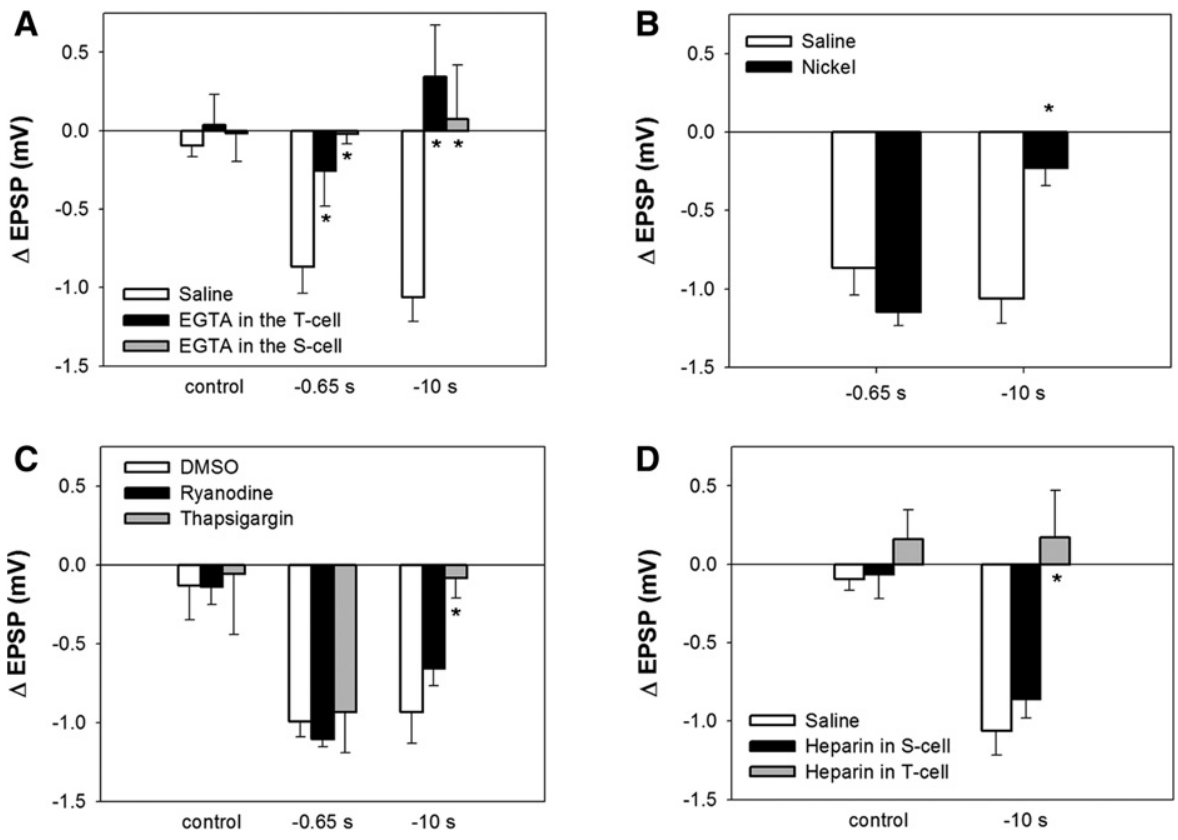

Figure 3. (A) EGTA injected into either the T or $S$ cell blocked LTD following both -0.65 -sec pairing and -10 -sec pairing. EGTA injections had no effect on EPSP amplitude when pairing was omitted (control). (B) Perfusion of $\mathrm{NiCl}_{2}$ (nickel) had no effect on LTD following -0.65 -sec pairing, but did block depression following -10 -sec pairing. (C) Perfusion of thapsigargin had no effect on LTD following -0.65 -sec pairing, but did block depression following $-10 \mathrm{sec}$ pairing. Perfusion of ryanodine had no effect on LTD following either -0.65 - or -10 -sec pairing. $(D)$ Injection of heparin into the T, but not the $S$ cell, blocked depression following -10-sec pairing. Heparin injection in the T or $S$ cell had no effect on EPSP amplitude when pairing was omitted (control). Asterisks indicate significant difference compared with vehicle control.

$P>0.05 ; n=5$ in each group). To further test the mechanisms by which store-released $\mathrm{Ca}^{2+}$ contributed to LTD, ryanodine $(50 \mu \mathrm{M})$, which inhibits ryanodine receptor (RyR)-mediated storereleased $\mathrm{Ca}^{2+}$, was perfused during pairing. Ryanodine had no effect on LTD following either -0.65 -sec or -10 -sec interval pairing (Fig. 3C; Newman-Keuls post hoc test, $P>0.05$ ), indicating that RyR-mediated store-released $\mathrm{Ca}^{2+}$ did not contribute to $\mathrm{T}$ to $S$ LTD. This result suggested that $\mathrm{Ca}^{2+}$ release from intracellular stores was mediated by inositol triphosphate (IP3) receptors; therefore, heparin was injected into either the T or S cell prior to -10 -sec interval pairing to block IP3 receptor-mediated $\mathrm{Ca}^{2+}$ release (Jin and Hawkins 2003). As shown in Figure 3D, injection of heparin $(2 \mathrm{mg} / \mathrm{mL})$ into the T cell, but not the $\mathrm{S}$ cell, blocked LTD following -10 -sec pairing (one-way ANOVA, $F_{(2,9)}=10.75$, $P<0.01$; Newman-Keuls post hoc test, $P<0.01 ; n=4$ in each group). Intracellular heparin injection in the absence of $T$ and $S$ cell pairing had no effect on synaptic transmission. These findings indicate that $\mathrm{Ca}^{2+}$ release from intracellular stores contributed to eCB-LTD, but not NMDAR-LTD and that store-released $\mathrm{Ca}^{2+}$ was mediated by IP3 receptors and not RyRs, consistent with earlier studies of eCB-LTD in the leech (Li and Burrell 2010).

\section{Role of protein phosphatases in timing-dependent LTD}

Both NMDAR- and eCB-LTD have been reported to require activation of protein phosphatases (Anwyl 2006; Heifets et al. 2008). To test the involvement of protein phosphatases in T to S NMDARand eCB-LTD, either $1 \mu \mathrm{M}$ okadaic acid, a specific inhibitor of protein phosphatases $1 / 2 \mathrm{~A}$, or $1 \mu \mathrm{M}$ cyclosporine $\mathrm{A}$, a phosphatase $2 \mathrm{~B}$ inhibitor, were applied via perfusion during pairing. As shown in Figure 4, both okadaic acid and cyclosporine A blocked LTD following -0.65-sec interval pairing (one-way ANOVA, $F_{(2,9)}=9.34, P<0.01$; Newman-Keuls post hoc test, $P<0.01$ okadaic acid vs. DMSO, $P<0.01$ cyclosporin A vs. DMSO; $n=4$ for each group), indicating that both types of phosphatases contributed to NMDAR-LTD. Both phosphatase inhibitors also blocked LTD following -10 -sec interval pairing (one-way ANOVA, $F_{(2,12)}=5.73, P<$ 0.05; Newman-Keuls post hoc test, $P<$ 0.05 okadaic acid vs. DMSO, $P<0.05$ cyclosporin A vs. DMSO; $n=5$ for each group), indicating that both phosphatases contributed to eCB-LTD. Neither phosphate inhibitor had any effect on the T to S EPSP when applied without pairing. These results are consistent with a number of earlier studies in the leech in which NMDAR- and endocannabinoid-dependent LTD were elicited with either pre-/postsynaptic pairing or LFS (Li and Burrell 2009; Grey and Burrell 2010; Yuan and Burrell 2010a).

\section{Discussion}

Using the $\mathrm{T}$ to $\mathrm{S}$ circuit in the leech, we have observed a novel pattern of Hebbian neuromodulation following the pairing of physiologically relevant spike trains. There have been relatively few studies in which trains or bursts of action potentials in both the pre- and postsynaptic neuron have been paired to produced synaptic plasticity (Lin and Glanzman 1997; Kobayashi and Poo 2004; Butts et al. 2007; Harnett et al. 2009; Grey and Burrell 2010) and only potentiation was observed in most cases regardless of the order of pre- and postsynaptic activity (the exceptions are Harnett et al. 2009; Grey and Burrell 2010). In the present study, the sign of synaptic change was sensitive to the order of the spike trains with $\mathrm{T}$ before $\mathrm{S}$ pairings producing LTP and $\mathrm{S}$ before $\mathrm{T}$ pairings producing LTD, similar to STDP. Induction of potentiation vs. depression also depended on the temporal proximity of the T- and S-cell activity, again consistent with STDP. However, the intervals between the T- and S-cell activity that supported potentiation or depression were much longer (hundreds of milliseconds to seconds) than the intervals that observed during STDP (tens of milliseconds).

Timing-dependent LTP was elicited by T before $S$ pairings of $0-1$ sec and was NMDAR-dependent. Interestingly, previous studies in which T to S LTP was elicited by tetanic stimulation found that potentiation was NMDAR-independent and instead required activation of mGluRs and PKC (Burrell and Sahley 2004; Burrell and Li 2008). It appears that different patterns of activity can elicit different forms of LTP in the T to S circuit, similar to what has been observed in the hippocampus (Hoffman et al. 2002).

The ability of different patterns of activity to elicit different mechanisms of neural plasticity was also observed during timingdependent LTD. NMDAR-LTD was observed following pairings with a relatively brief $S$ before $T$ interval $(-0.65 \mathrm{sec})$, whereas eCB-LTD was observed following pairing with a longer pre-post interval $(-10 \mathrm{sec})$. LTD following pairing with an interval of $-1 \mathrm{sec}$ appeared to utilize both NMDA receptor- and cannabinoid-dependent signaling pathway. These results parallel an earlier study of T to S LTD in which LFS applied for $450 \mathrm{sec}$ elicited 


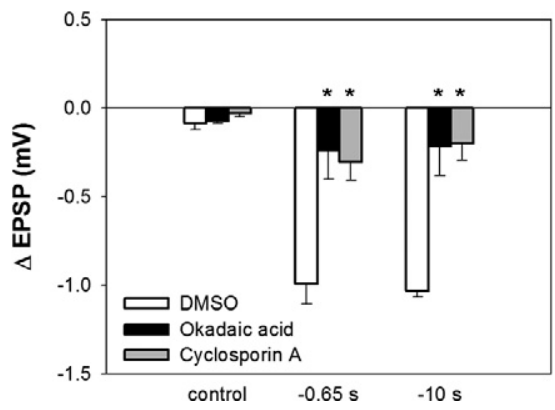

Figure 4. Perfusion of okadaic acid or cyclosporine A significantly attenuated LTD following both -0.65 - and -10 -sec pairing. Neither drug altered EPSP amplitude when pairing was omitted (control). Asterisks indicate significant difference compared with vehicle control.

NMDAR-LTD, whereas LFS applied for 900 sec elicited eCB-LTD (Li and Burrell 2009).

\section{Properties of NMDAR-LTD}

NMDAR-LTD in the $\mathrm{T}$ to $\mathrm{S}$ pathway appeared to require both NMDAR activation and an increased intracellular $\mathrm{Ca}^{2+}$ in the $\mathrm{S} / \mathrm{C}$ cell, based on the experiments in which the injection of MK-801 or EGTA into the S-cell blocked depression. NMDARLTD was also blocked by inhibitors of both protein phosphatase $1 / 2 \mathrm{~A}$ and $2 \mathrm{~B}$, consistent with earlier studies of LFS-induced depression in this circuit (Li and Burrell 2009). EGTA injection into the T cell also prevented NMDAR-LTD. This is a somewhat surprising result given that the source of increased $\mathrm{Ca}^{2+}$ levels in the $\mathrm{T}$ cell is unknown. Injection of MK-801 into the T cell did not affect LTD following -0.65 -sec pairing indicating that $\mathrm{T}$ cell NMDARs are not involved. Furthermore, inhibitors of VGCCs and $\mathrm{Ca}^{2+}$ release from intracellular stores also had no effect on NMDAR-LTD. One possible explanation is that this $\mathrm{Ca}^{2+}$ signal is mediated by high voltage-activated (HVA) $\mathrm{Ca}^{2+}$ channels. The concentration of $\mathrm{NiCl}_{2}$ used to block VGCCs in the present study is effective on low voltage-activated (LVA) $\mathrm{Ca}^{2+}$ channels in the leech, but not on HVA $\mathrm{Ca}^{2+}$ channels (Ivanov and Calabrese 2006a).

\section{Properties of eCB-LTD}

eCB-LTD involved postsynaptic synthesis of 2-AG, based on the observation that depression was blocked following injection of the DAG lipase inhibitor THL into the $\mathrm{S}$ cell. These results agree with previous studies in which eCB-LTD in a leech nociceptive synapse was prevented by inhibition of postsynaptic 2-AG synthesis (Yuan and Burrell 2010a). Endocannabinoid transmitters are evolutionarily well conserved, with 2-AG being the most abundant endocannabinoid in the CNS of both vertebrates and invertebrates (Matias et al. 2001; Elphick and Egertova 2005; Lehtonen et al. 2008; Leung et al. 2008). Furthermore, endocannabinoiddependent synaptic modulation has been observed in invertebrate nervous systems (Lemak et al. 2007; Li and Burrell 2009, 2010; Yuan and Burrell 2010a).

It is proposed that $2 \mathrm{AG}$ travels in a retrograde manner to the presynaptic neurons where it binds to a TRPV-like receptor (Fig. 5A). This conclusion is based on experimental data in the present study in which the TRPV receptor antagonist capsazepine prevented LTD when bath-applied or when injected into the T cell, but not the $S$ cell. These results are consistent with a recent study by Yuan and Burrell (2010), which used both capsazepine and a more selective TRPV1 antagonist to block eCB-LTD. Both presynaptic and postsynaptic TRPV1 receptors have been found to mediate eCB-LTD in the mammalian CNS (Gibson et al. 2008; Chavez et al. 2010; Grueter et al. 2010).

eCB-LTD requires increases in intracellular $\mathrm{Ca}^{2+}$ in both the $\mathrm{T}$ and $\mathrm{S}$ cells and there are multiple sources for this $\mathrm{Ca}^{2+}$ signal.
A

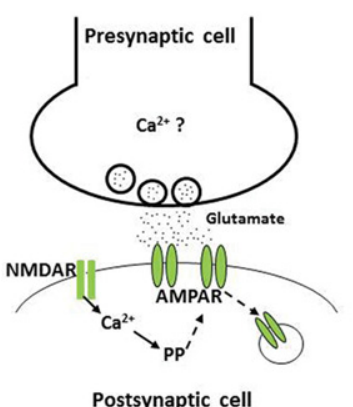

eCB-LTD

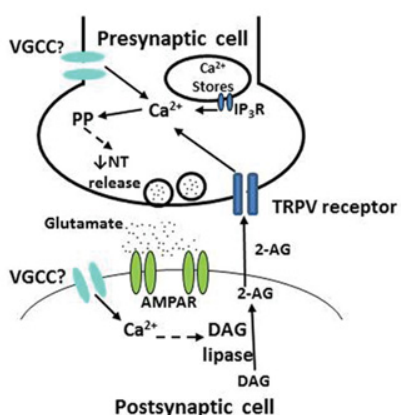

B

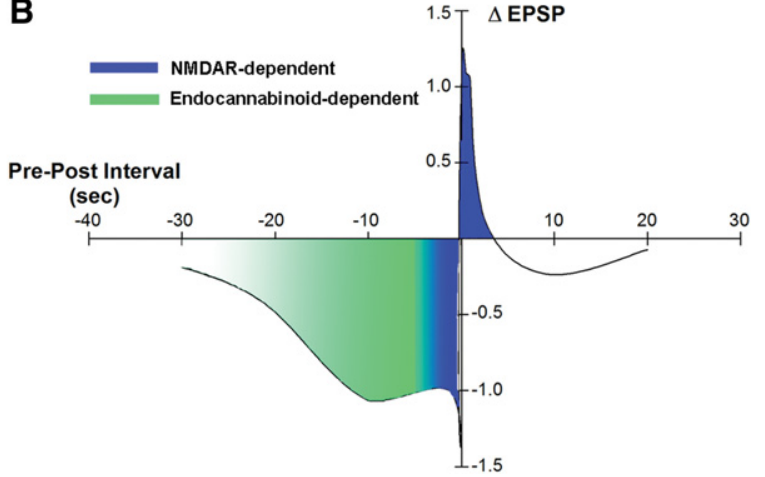

Figure 5. (A) Proposed cellular mechanisms mediating NMDAR-LTD and eCB-LTD. During NMDR-LTD activation of postsynaptic (S/C cell) NMDARs elicits an influx of $\mathrm{Ca}^{2+}$ that then triggers protein phosphatases (PP). PP activity is hypothesized (indicated by dashed arrows) to mediate removal of synaptic AMPA receptors (AMPAR). This study also found that an increase in presynaptic intracellular $\mathrm{Ca}^{2+}$ was also required for NMDAR-mediated LTD, but the source of this $\mathrm{Ca}^{2+}$ signal and its functional role are not known at this time (indicated by "?"). During eCB-LTD postsynaptic DAG lipase converts DAG into 2-AG. This process is potentially $\mathrm{Ca}^{2+}$-dependent (indicated by the dashed arrow) with VGCCs being the likely source of postsynaptic Ca ${ }^{2+}$ (the "?" next to the VGCC indicates that it is unknown whether these channels operate presynaptically, postsynaptically, or both). 2-AG travels in a retrograde

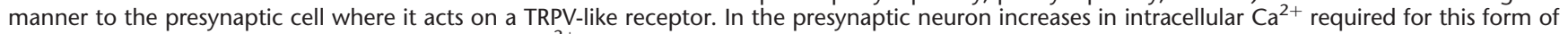
depression are likely the result of IP3R-mediated $\mathrm{Ca}^{2+}$ release from intracellular stores, influx through the activated TRPV-like receptor and possibly VGCCs. The resulting $\mathrm{Ca}^{2+}$ signal activates protein phosphatases that mediate a decrease in neurotransmitter release. Because of the polysynaptic nature of this circuit (see Fig. 1A), presynaptic events may be occurring in either the T cell or the unknown interneuron between the T and S/C cells. The most likely location of postsynaptic events is in the C cell itself. (B) Proposed relationship between the temporal pattern of T- and S-cell pairing and the cellular mechanisms engaged to mediate changes in signaling. NMDAR-dependent processes (blue) are initiated when the interval between T- and S-cell activity is $1 \mathrm{sec}$ or less, with LTP observed following T before S pairings (positive pre-post intervals) and LTD observed following S before T pairings (negative intervals). Endocannabinoid-dependent processes (green) are initiated when $S$ before T pairing intervals are $>-1$ sec and always produce LTD. There may be pairing interval range (between -1 and $-10 \mathrm{sec}$ ) depression transitions from NMDAR- to endocannabinoid-mediated LTD, but endocannabinoid-dependent processes alone mediated depression when the pairing interval is $-10 \mathrm{sec}$. 
Influx of extracellular $\mathrm{Ca}^{2+}$ via VGCCs was one source, although it was impossible to determine whether these channels were located. Presynaptic TRPV-like receptors are likely a second source of $\mathrm{Ca}^{2+}$ influx contributing to eCB-LTD. $\mathrm{Ca}^{2+}$ release from intracellular stores mediated by IP3, but not ryanodine receptors, also contributed to eCB-LTD. Similar to earlier studies of the leech (Li and Burrell 2009), both okadaic acid and cyclosporine A inhibited eCB-LTD, indicating the potential involvement of multiple types of protein phosphatases during this form of depression. A tentative model of the mechanisms mediating NMDAR- vs. eCB-LTD is presented in Figure 5.

It is acknowledged that because the $\mathrm{T}$ to $\mathrm{S}$ connection is a polysynaptic circuit, it is impossible to know the exact properties of the unknown interneurons between the $\mathrm{T}$ and $\mathrm{S}$ cells during LTP or LTD. Nevertheless, pairing T-cell and S-cell activity elicited a reproducible pattern of plasticity indicating that there was sufficient control of these unknown interneurons by T-cell stimulation. It should also be pointed out that functionally relevant activity of the unknown interneurons would require activation of the T cell, justifying a study of the plasticity across the entire polysynaptic circuit. Injections of several drugs into either the $\mathrm{T}$ cell or the $\mathrm{S}$ cell were effective in blocking LTD despite the polysynaptic nature of this circuit. In the case of the T cell, it is possible that drugs injected into the $T$ cell have access to the unknown interneurons, potentially by traveling through gap junctions linking these cells, similar to the $S$ and $C$ cells (to date, all reported synapses made by the $\mathrm{T}$ cell have a gap junction-mediated component) (Baylor and Nicholls 1969; Nicholls and Purves 1970; Muller and Scott 1981; Velazquez-Ulloa et al. 2003; Li and Burrell 2008). The ability of T-cell-injected drugs to block plasticity indicates that modulation may occur between the $\mathrm{T}$ and unknown interneuron, or between the unknown interneuron and the $\mathrm{S}$ cell or both. In the case of the S cell, it is likely that the injected drugs travel from the $S$ cell to the electrically coupled C cells (Fig. 1A). The majority of synaptic input by the $\mathrm{T}$ cell to the $\mathrm{S}$ interneuron is through input to the $\mathrm{C}$ cells and small molecular weight dyes and pharmacological agents have been shown to spread from the S cell to the C cell (Muller and Scott 1981; Li and Burrell 2010).

\section{Pairing of spike trains elicits a complex pattern of neural plasticity}

Although there are examples of STDP using distinct cellular mechanisms to mediate LTP vs. LTD (Bender et al. 2006; Nevian and Sakmann 2006; Fino et al. 2010), what is unique in the T to $S$ circuit is that both NMDAR- and endocannabinoid-dependent processes mediate LTD, but each appears to be activated within a distinct range of pairing intervals (Fig. 5B). NMDARs appear to be activated selectively when the T-S pairing interval is $\leq-1 \mathrm{sec}$, similar to the temporal window observed during LTP and is consistent with the well-established role of NMDARs detecting the coincidence between presynaptic glutamate release and postsynaptic depolarization (Nowak et al. 1984; Caporale and Dan 2008). Endocannabinoid (2-AG) synthesis appears to be selectively activated when the T-S pairing interval is -1 to $-10 \mathrm{sec}$. This result is similar to eCB-LTD observed in hippocampal inhibitory synapses in which a depression was elicited when postsynaptic release of endocannabinoids was followed by presynaptic activity within minutes of the cannabinoid signal (Heifets et al. 2008). The authors of that study proposed that presynaptic calcineurin (protein phosphatase 2B) detects the coincidence of the endocannabinoid signaling and presynaptic activity, and calcineurin was also found to be required for eCB-LTD in both the $\mathrm{T}$ to $\mathrm{S}$ pathway and in the monosynaptic connection between nociceptive sensory neurons and the motor neurons in the leech (Yuan and Burrell 2010b).
That the pairing of spike trains may result in plasticity that has properties distinct from STDP is not surprising when one considers the ability of spike trains to initiate cellular signaling processes that would not be initiated by a single action potential (Lisman 1997). This may provide a physiological basis for the prediction by Stent (1973) that the amount of activity, in terms of either the duration or frequency of spikes that make up the train, is a critical element in determining the properties of associative synaptic plasticity. In addition, using spike trains may expand the temporal window during which coordinated pre- and postsynaptic activity can elicit LTP and LTD, a finding that is consistent with the results in this study. This ability to expand the temporal window that will support Hebbian plasticity in neural circuits may be critical in having synaptic level events translated into behavioral changes during classical conditioning, where the interval between learned stimuli can be much greater than supported by STDP (Drew and Abbott 2006).

\section{Material and Methods}

\section{Animal and drug preparation}

Leeches (3 g) were obtained from a commercial supplier (Leeches USA, Westbury, NY) and kept in pond water $\left(0.52 \mathrm{~g} / \mathrm{L} \mathrm{H}_{2} \mathrm{O}\right.$ Hirudo salt [Leeches USA Ltd.]) on a 12-h light/dark daily cycle at $15^{\circ} \mathrm{C}$. All the dissections and recordings were carried out in leech saline containing: $110 \mathrm{mM} \mathrm{NaCl}, 4 \mathrm{mM} \mathrm{KCl}, 1.8 \mathrm{mM} \mathrm{CaCl}_{2}, 1 \mathrm{mM}$ $\mathrm{MgCl}_{2}, 5 \mathrm{mM} \mathrm{NaOH}$, and $10 \mathrm{mM}$ HEPES. Okadaic acid (Tocris), cyclosporine A (Tocris), tetrahydrolipstatin (THL; Sigma), 1-(2,4Dichlorophenyl)-5-(4-iodophenyl)-4-methyl - $N$-1-piperidinyl-1Hpyrazole-3-carboxamide (AM251; Tocris), $O, O^{\prime}-[1,6$-Hexanediyl bis(iminocarbonyl)] dioxime cyclohexanone (RHC80267; Tocris), ryanodine (Tocris), thapsigargin (Tocris), and capsazepine (Tocris) were stocked in DMSO. Ethylene glycol tetra-acetic acid (EGTA; Sigma), 2-Amino-5-phosphonopentanoic acid (AP5; Sigma), (5R,10S)-(+)-5-Methyl-10,11-dihydro-5-H-dibenzo[a,d] cyclohepten-5,10-imine (MK-801; Sigma) and heparin (Sigma) were stocked in saline. Final concentrations of okadaic acid and cyclosporine A were prepared in saline and made just prior to the start of individual experiments. For intracellular iontophoretic injection, MK801 (Tocris), heparin, and EGTA (Sigma) were dissolved in $3 \mathrm{M}$ KAc. Final concentrations of THL and capsazepine were prepared with $3 \mathrm{M}$ KAc before the start of individual experiments. Final DMSO concentration for okadaic acid, cyclosporin $\mathrm{A}, \mathrm{THL}$, and capsazepine were $0.005 \%, 0.005 \%, 0.02 \%$, and $0.5 \%$, respectively. A total of $1 \mathrm{mM} \mathrm{NiCl}_{2}$ solution was freshly prepared in saline before each experiment.

\section{Electrophysiology}

A chain of two midbody ganglia was dissected and placed in a recording chamber with suction electrodes applied to the anterior nerve root and the posterior end of the connective nerve to stimulate the $\mathrm{T}$ cells and the $\mathrm{S}$ cell, respectively, during pairing (Supplemental Fig. 1A). Stimulus pulses were delivered using a digital programmable stimulator (Multi-Channel Systems STG 1004) through an AC/DC differential amplifier (A-M systems Inc., Model 3000). Stimulus intensity was set at the minimum to activate the $\mathrm{T}$ and $\mathrm{S}$ cells, which have the largest axons and therefore the lowest thresholds in their respective nerves. Intracellular recordings from P cells during anterior nerve stimulation prior to $\mathrm{T} / \mathrm{S}$ pairing confirmed these cells were not activated by anterior nerve stimulation (data not shown). Eliciting activity in this manner was chosen because it more closely resembles the manner in which spike trains are initiated and travel through these neurons compared with current injection in the soma. It is possible that this nerve stimulation activates other neurons, either directly or synaptically, that might contribute to the observed plasticity. However, changes in the T to S EPSP appeared to correspond to the interval between the T- and S-cell spike trains, and it was possible to block this plasticity using drugs 
selectively injected into either the $\mathrm{T}$ or $\mathrm{S}$ cells (see Results). Therefore, it seems unlikely that changes in the other, unknown neurons contribute to the observed plasticity.

During pairing, the stimuli applied to the anterior nerve of first ganglion consisted of five 1 -msec nerve shocks at $10 \mathrm{~Hz}$. The connective nerve of second ganglion received $201-\mathrm{msec}$ shocks at $25 \mathrm{~Hz}$ (Supplemental Fig. 1B), a level of activity that corresponds to the S-cell response to a strong mechanosensory stimulation (Burrell and Sahley 2005). Different intervals between the T- and S-cell spike trains were tested and the intervals were calculated as the time between the start of T-cell train and the start of the S-cell postsynaptic train. The T-/S-cell pairings were repeated 10 times with a 2-min intertrial interval (ITI) (Supplemental Fig. 1B). Intracellular recordings of the $T$ and $S$ cell were made prior to (pretest) and $1 \mathrm{~h}$ after (post-test) pairing to measure changes in the T to S EPSP.

Dual electrode recordings to measure changes in the T to $\mathrm{S}$ EPSP were carried out as described previously (Li and Burrell 2009). Briefly, recordings were performed by impaling neurons with sharp glass microelectrodes using a manual micropositioner (Model 1480; Siskiyou Inc.). Microelectrodes were pulled from borosilicate capillary tubing $(1.0 \mathrm{~mm}$ o.d., $0.75 \mathrm{~mm}$ i.d.; FHC) to a resistance of $40-50 \mathrm{M} \Omega$ and filled with $3 \mathrm{M}$ potassium acetate. Current pulses were delivered to the neurons using a digital programmable stimulator (Multi-Channel Systems STG 1004). Signals were amplified using a bridge amplifier (BA-1S; NPI) and then digitally converted (Digidata $1322 \mathrm{~A} A$ /D converter) for viewing and subsequent analysis (Axoscope 9.0; Clampfit 9.0). During the pre- and post-tests, S-cell input resistance was monitored and only experiments with consistent input resistance were included in further data analysis. Across all experiments, pre- and post-test test input resistance for the $\mathrm{S}$ cell was $15.6 \pm 0.2 \mathrm{M} \Omega$ and $14.4 \pm$ $0.6 \mathrm{M} \Omega$, respectively.

In experiments with intracellular drugs delivery, an iontophoretic protocol was applied to either T or S cells. Under these conditions, drug stocks were dissolved in $3 \mathrm{M}$ KAc to make final concentration. The iontophoretic protocol consisted of $-1 \mathrm{nA}$, $500-\mathrm{msec}$ current pulse delivered at $1 \mathrm{~Hz}$ for $5 \mathrm{~min}$ prior to the pairing protocol. Previous experiments (Li and Burrell 2010) have shown that drugs injected into the $S$ cell will enter the $C$ cells where the majority of synaptic input reaching the $\mathrm{S}$ cell is located (Muller and Scott 1981).

\section{Statistics}

For each experimental treatment only one ganglion was used from an individual animal; that is, the sample size for a given treatment reflects the number of ganglia and the number of animals. $\triangle \mathrm{EPSP}$ were calculated as $\mathrm{EPSP}_{\text {post }}-\mathrm{EPSP}_{\text {pre }}$ and presented as mean $\pm \mathrm{SE}$. Statistical analyses were performed using one-way ANOVA and post hoc Newman-Keuls test with Statistica analysis software (Statsoft).

\section{Acknowledgments}

The authors thank Drs. Pasquale Manzera, Gina Forster, Doug Martin, Joyce Keifer, and two anonymous reviewers for helpful comments during the preparation of this manuscript. This work was supported by a subproject of the National Institutes of Health grant (P20 RR-015567; J Keifer, PI), which is designated as a Center of Biomedical Research Excellence (COBRE).

\section{References}

Anwyl R. 2006. Induction and expression mechanisms of postsynaptic NMDA receptor-independent homosynaptic long-term depression. Prog Neurobiol 78: 17-37.

Baylor DA, Nicholls JG. 1969. Chemical and electrical synaptic connexions between cutaneous mechanoreceptor neurones in the central nervous system of the leech. J Physiol 203: 591-609.

Bender VA, Bender KJ, Brasier DJ, Feldman DE. 2006. Two coincidence detectors for spike timing-dependent plasticity in somatosensory cortex. J Neurosci 26: 4166-4177.
Burrell BD, Li Q. 2008. Co-induction of long-term potentiation and long-term depression at a central synapse in the leech. Neurobiol Learn Mem 90: 275-279.

Burrell BD, Sahley CL. 2004. Multiple forms of long-term potentiation and long-term depression converge on a single interneuron in the leech CNS. J Neurosci 24: 4011-4019.

Burrell BD, Sahley CL. 2005. Serotonin mediates learning-induced potentiation of excitability. J Neurophysiol 94: 4002-4010.

Burrell BD, Sahley CL, Muller KJ. 2003. Progressive recovery of learning during regeneration of a single synapse in the medicinal leech. J Comp Neurol 457: $67-74$.

Butts DA, Kanold PO, Shatz CJ. 2007. A burst-based “Hebbian" learning rule at retinogeniculate synapses links retinal waves to activity-dependent refinement. PLoS Biol 5: e61. doi: 10.1371/journal.pbio.0050061.

Caporale N, Dan Y. 2008. Spike timing-dependent plasticity: A Hebbian learning rule. Annu Rev Neurosci 31: 25-46.

Chavez AE, Chiu CQ, Castillo PE. 2010. TRPV1 activation by endogenous anandamide triggers postsynaptic long-term depression in dentate gyrus. Nat Neurosci 13: 1511-1518.

Crisp KM, Burrell BD. 2008. Cellular and behavioral properties of learning in the leech and other annelids. In Annelids as model systems in the biological sciences (ed. D Shain). John Wiley \& Sons, New York.

Di Marzo V, Bisogno T, De Petrocellis L, Melck D, Martin BR. 1999. Cannabimimetic fatty acid derivatives: The anandamide family and other endocannabinoids. Curr Med Chem 6: 721-744.

Di Marzo V, Bisogno T, De Petrocellis L. 2001. Anandamide: Some like it hot. Trends Pharmacol Sci 22: 346-349.

Drew PJ, Abbott LF. 2006. Extending the effects of spike-timing-dependent plasticity to behavioral timescales. Proc Natl Acad Sci 103: 8876-8881.

Elphick MR, Egertova M. 2005. The phylogenetic distribution and evolutionary origins of endocannabinoid signalling. Handb Exp Pharmacol 168: 283-297.

Fino E, Paille V, Cui Y, Morera-Herreras T, Deniau JM, Venance L. 2010. Distinct coincidence detectors govern the corticostriatal spike timing-dependent plasticity. J Physiol 588: 3045-3062.

Gibson HE, Edwards JG, Page RS, Van Hook MJ, Kauer JA. 2008. TRPV1 channels mediate long-term depression at synapses on hippocampal interneurons. Neuron 57: 746-759.

Golech SA, McCarron RM, Chen Y, Bembry J, Lenz F, Mechoulam R, Shohami E, Spatz M. 2004. Human brain endothelium: Coexpression and funtion of vanilloid and endocannabinoid receptors. Mol Brain Res 132: $87-92$.

Grey KB, Burrell BD. 2010. Co-induction of LTP and LTD and its regulation by protein kinases and phosphatases. J Neurophysiol 103: 2737-2746.

Grey KB, Moss BL, Burrell BD. 2009. Molecular identification and expression of the NMDA receptor NR1 subunit in the leech. Invert Neurosci 9: 11-20.

Grueter BA, Brasnjo G, Malenka RC. 2010. Postsynaptic TRPV1 triggers cell type-specific long-term depression in the nucleus accumbens. Nat Neurosci 13: 1519-1525.

Harnett MT, Bernier BE, Ahn KC, Morikawa H. 2009. Burst-timingdependent plasticity of NMDA receptor-mediated transmission in midbrain dopamine neurons. Neuron 62: 826-838.

Hebb DO. 1949. The organization of behavior; a neuropsychological theory. Wiley, New York.

Heifets BD, Chevaleyre V, Castillo PE. 2008. Interneuron activity controls endocannabinoid-mediated presynaptic plasticity through calcineurin. Proc Natl Acad Sci 105: 10250-10255.

Hoffman DA, Sprengel R, Sakmann B. 2002. Molecular dissection of hippocampal theta-burst pairing potentiation. Proc Natl Acad Sci 99: 7740-7745.

Ivanov AI, Calabrese RL. 2006a. Graded inhibitory synaptic transmission between leech interneurons: Assessing the roles of two kinetically distinct low-threshold Ca currents. I Neurophysiol 96: 218-234.

Ivanov AI, Calabrese RL. 2006b. Spike-mediated and graded inhibitory synaptic transmission between leech interneurons: Evidence for shared release sites. J Neurophysiol 96: 235-251.

Jin I, Hawkins RD. 2003. Presynaptic and postsynaptic mechanisms of a novel form of homosynaptic potentiation at aplysia sensory-motor neuron synapses. J Neurosci 23: 7288-7297.

Kobayashi K, Poo MM. 2004. Spike train timing-dependent associative modification of hippocampal CA3 recurrent synapses by mossy fibers. Neuron 41: $445-454$.

Kristan WB Jr, Calabrese RL, Friesen WO. 2005. Neuronal control of leech behavior. Prog Neurobiol 76: 279-327.

Lehtonen M, Reisner K, Auriola S, Wong G, Callaway JC. 2008. Mass-spectrometric identification of anandamide and 2-arachidonoylglycerol in nematodes. Chem Biodivers 5: 2431-2441.

Lemak MS, Bravarenko NI, Bobrov MY, Bezuglov VV, Ierusalimsky VN, Storozhuk MV, Malyshev AY, Balaban PM. 2007. Cannabinoid regulation in identified synapse of terrestrial snail. Eur J Neurosci 26: 3207-3214. 
Leung HT, Tseng-Crank J, Kim E, Mahapatra C, Shino S, Zhou Y, An L, Doerge RW, Pak WL. 2008. DAG lipase activity is necessary for TRP channel regulation in Drosophila photoreceptors. Neuron 58: 884-896.

Li Q, Burrell BD. 2008. CNQX and AMPA inhibit electrical synaptic transmission: A potential interaction between electrical and glutamatergic synapses. Brain Res 1228: 43-57.

Li Q, Burrell BD. 2009. Two forms of long-term depression in a polysynaptic pathway in the leech CNS: One NMDA receptor-dependent and the other cannabinoid-dependent. J Comp Physiol A 195: $831-841$.

Li Q, Burrell BD. 2010. Properties of cannabinoid-dependent long term depression in the leech. J Comp Physiol A Neuroethol Sens Neural Behav Physiol 196: 841-851.

Lin XY, Glanzman DL. 1997. Effect of interstimulus interval on pairing-induced LTP of Aplysia sensorimotor synapses in cell culture. J Neurophysiol 77: 667-674.

Lisman JE. 1997. Bursts as a unit of neural information: Making unreliable synapses reliable. Trends Neurosci 20: $38-43$.

Maione S, Cristino L, Migliozzi AL, Georgiou AL, Starowicz K, Salt TE, Di Marzo V. 2009. TRPV1 channels control synaptic plasticity in the developing superior colliculus. J Physiol 587: 2521-2535.

Matias I, Bisogno T, Melck D, Vandenbulcke F, Verger-Bocquet M, De Petrocellis L, Sergheraert C, Breton C, Di Marzo V, Salzet M. 2001. Evidence for an endocannabinoid system in the central nervous system of the leech Hirudo medicinalis. Brain Res Mol Brain Res 87: $145-159$.

McPartland JM, Agraval J, Gleeson D, Heasman K, Glass M. 2006. Cannabinoid receptors in invertebrates. J Evol Biol 19: 366-373.

Modney BK, Sahley CL, Muller KJ. 1997. Regeneration of a central synapse restores nonassociative learning. J Neurosci 17: 6478-6482.

Muller KJ, Scott SA. 1981. Transmission at a "direct" electrical connexion mediated by an interneurone in the leech. J Physiol 311: 565-583.

Nevian T, Sakmann B. 2006. Spine Ca2 + signaling in spike-timingdependent plasticity. J Neurosci 26: 11001-11013.

Nicholls JG, Purves D. 1970. Monosynaptic chemical and electrical connexions between sensory and motor cells in the central nervous system of the leech. J Physiol 209: 647-667.
Nowak L, Bregestovski P, Ascher P, Herbet A, Prochiantz A. 1984. Magnesium gates glutamate-activated channels in mouse central neurones. Nature 307: 462-465.

Ortar G, Bisogno T, Ligresti A, Morera E, Nalli M, Di Marzo V. 2008. Tetrahydrolipstatin analogues as modulators of endocannabinoid 2-arachidonoylglycerol metabolism. J Med Chem 51: 6970-6979.

Qin N, Neeper MP, Liu Y, Hutchinson TL, Lubin ML, Flores CM. 2008. TRPV2 is activated by cannabidiol and mediates CGRP release in cultured rat dorsal root ganglion neurons. J Neurosci $\mathbf{2 8 :}$ $6231-6238$.

Rawls SM, Gomez T, Raffa RB. 2007. An NMDA antagonist (LY 235959) attenuates abstinence-induced withdrawal of planarians following acute exposure to a cannabinoid agonist (WIN 52212-2). Pharmacol Biochem Behav 86: 499-504.

Sahley CL, Modney BK, Boulis NM, Muller KJ. 1994. The S cell: An interneuron essential for sensitization and full dishabituation of leech shortening. J Neurosci 14: 6715-6721.

Shaw BK, Kristan WB Jr. 1995. The whole-body shortening reflex of the medicinal leech: Motor pattern, sensory basis, and interneuronal pathways. J Comp Physiol [A] 177: 667-681.

Stella N, Schweitzer P, Piomelli D. 1997. A second endogenous cannabinoid that modulates long-term potentiation. Nature 388: 773-778.

Stent GS. 1973. A physiological mechanism for Hebb's postulate of learning. Proc Natl Acad Sci 70: 997-1001.

Velazquez-Ulloa N, Blackshaw SE, Szczupak L, Trueta C, Garcia E, De Miguel FF. 2003. Convergence of mechanosensory inputs onto neuromodulatory serotonergic neurons in the leech. J Neurobiol 54: $604-617$.

Yuan S, Burrell BD. 2010a. Endocannabinoid-dependent LTD in a nociceptive synapse requires activation of a presynaptic TRPV-like receptor. J Neurophysiol 104: 2766-2777.

Yuan S, Burrell BD. 2010b. LTD in a nociceptive synapse requires postsynaptic endocannabinoid synthesis and presynaptic TRPV receptor activation. In 2010 Neuroscience meeting planner, abstract program no. 281.14. Society for Neuroscience, San Diego, CA.

Received April 22, 2011; accepted in revised form June 29, 2011. 


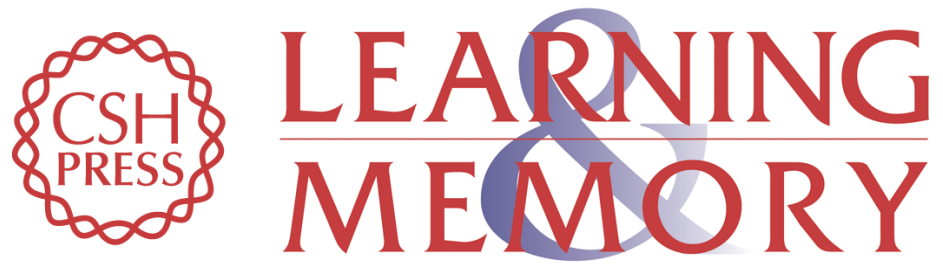

\section{Associative, bidirectional changes in neural signaling utilizing NMDA receptor- and endocannabinoid-dependent mechanisms}

Qin Li and Brian D. Burrell

Learn. Mem. 2011, 18:

Access the most recent version at doi:10.1101//m.2252511 Supplemental http://learnmem.cshlp.org/content/suppl/2011/08/08/18.9.545.DC1
Material

References This article cites 53 articles, 11 of which can be accessed free at: http://learnmem.cshlp.org/content/18/9/545.full.html\#ref-list-1

License

Email Alerting Receive free email alerts when new articles cite this article - sign up in the box at the Service top right corner of the article or click here. 